\title{
POSSIBLE ALLELOPATHIC EFFECT OF FIVE SELECTED WEED SPECIES ON GERMINATION AND SEEDLING GROWTH OF WHEAT
}

\author{
Osman, M. S. E.; E. A. El-Ghareib*; K. M. Ghanem ${ }^{* *}$ and \\ A. A. El-Sheshtawy ${ }^{*}$ \\ * Agronomy Dept., Fac. Agric., Al-Azhar Univ., Cairo, Egypt. \\ ** Environment and Bio-Agric. Dept., Fac. Agric., Al-Azhar Univ., Cairo, Egypt

\section{ABSTRACT}

These experiments were performed in Agronomy Department, Faculty of Agriculture, Al-Azhar University, during 2008/2009 and 2009/2010 seasons to investigate the allelopathic potential effect of five weed species viz. Chenopodium album, L. $\left(\mathrm{W}_{1}\right)$, Euphorbia peplus, L. ( $\left.\mathrm{W}_{2}\right)$, Melilotus indica, (L.) All. $\left(\mathrm{W}_{3}\right)$, Avena fatua, L. $\left(\mathrm{W}_{4}\right)$ and Phalaris minor, Retz. $\left(\mathrm{W}_{5}\right)$ on germination and seedling development of wheat (Triticum aestivum, L. local var. Sakha 94). So,two experiments were carried out, the first (in laboratory) to study the effect of weed parts (root and shoot) water extract at different concentration $(0,10,20$ and $30 \%)$. The second experiment (in wirehouse) to study the effect of previous weed shoot residues at different concentration $(0,1,2$ and $3 \%(w / w))$. Results of the first experiment indicated that the differences between weed species for all studied traits were significant in the means of two seasons. All extracted of Chenopodium album, L. and Melilotus indica, (L.) All. significantly inhibited germination \%, germination rate, root length, shoot length, root and shoot dry weights compared with other weed species. The differences between weed parts extracted were significant for all studied traits in the means of two seasons. Shoot extraction significantly inhibited all studied traits. Also, results indicated that the differences between extract concentrations were significant for all studied attributed in the means of the two seasons. Application 30\% concentration significantly decreased grain germination and seedling growth of wheat. All the interactions between weed species, weed parts and concentration extracted were significant, except germination rate in the means of two seasons. Melilotus indica, (L.) All. $\times$ the shoot extract $\times 30 \%$ concentration increased effective allelopathic on all studied attributes under experiment conditions.

Results of the second experiment indicated that the effects of weed residues species and concentrations on seedling growth of wheat were significant in means of two seasons. The interactions between different species and 3\% concentration were significant for all studied traits. Avena fatua, L. and $3 \%$ concentration significantly inhibited most studied traits in means of two seasons.

Keywords: Allelopathy, aqueous extract, weed residues, weed species, weed parts, germination, seedling growth, wheat, Chenopodium album, L., Euphorbia peplus, L., Melilotus indica, (L.) All., Avena fatua, L. and Phalaris minor, Retz.

\section{INTRODUCTION}

Wheat (Triticum aestivum, L.) is the most grown crop in the world and has an economical significance for the humankind (Löve 1984). In Egypt the area of wheat was estimated at about $3.15^{\text {(1) }}$ million faddan (2) in 2008/2009 season, which produced about 8.52 million tons. Among many factors, which adversely influence the crop yield, weed infestation is the devastating one.

(1) Ministry of Agriculture and Land Reclamation, Agriculture Stats.

(2) Faddan $=4200.78 \mathrm{~m}^{2}$ 
Osman, M. S. E. et al.

Weed is a serious pest that damages most of crops; moreover it is everlasting problem for our agriculture. Weeds present in wheat fields that compete with crop plants for light, moisture and other essential nutrients and increase the cost of production. The reduction of wheat yield due to weed infestation amounted $30 \%$ (Nisha et al. 1999) or $61 \%$ (Hucl, 1998) as compared to weed free control. Moreover, weeds can influence wheat crop, by allelopathy which is usually harmful (Boonitiee and Ritdhit, 1984). Rice (1974) defined allelopathy as any direct or indirect harmful effect by one plant to another through the production of chemical compounds, which escape into the environment. Allelopathic substances are most commonly found in plant extracts and in plant residues of soil, some were found in live plant exudates and as volatile gases liberated from leaves and rhizome (Keeley, 1987).

Residues and leachate of several weeds have been shown to possess negative impacts on wheat growth and yield. Bhowmik and Doll (1982) studied the response of corn and soybean to various weed residues included Chenopodium album and demonstrated that plant height and shoot or root fresh weight of both corn and soybean were decreased as Chenopodium album residue concentration increased into soil. They suggested that these effects may be due to allelochemical such as phenolic acids including $p$ coumaric acid and other natural substances in the residues. Qasem (1993 a $\&$ b) studied the allelopathic effects of many weed species on wheat and found that the water extracts of shoots and roots inhibited; seed germination, coleoptile and root length as well as their dry weight. The inhibition rate of shoot and root extract were $88 \%$ and $20 \%$,respectively, moreover, the decayed residues of weeds delayed the emergence of wheat. Mallik et al. (1994) tested aqueous extract of air dried lambsquarters (Chenopodium album) on wheat. They found that the extract significantly inhibited seed germination and seedling growth of wheat. El-Khatib et al. (2004) reported that Chenopodium murale root and shoot aqueous extracts reduced seed germination, seedling establishment, plant growth and metabolite production of wheat (Triticum pyramidal). Leaf area and dry matter production showed a decreasing trend in response to the different treatments. Similar effects were found for pigment, carbohydrate and protein contents. In general, inhibition percentage was a function of extract concentration and plant tissue type. Shoot treatment was more strongly inhibitory than root treatment. The target species arranged from the most affected to the least affected were Melilotus indicus - Trifolium alexandrinum - Triticum pyramida I- Lycopersicon esculentum - Cucumis sativus.

Kaushalya et al. (2005) found that root aqueous extract of oat (Avena sativa) up to $5 \%$ concentration increased the radical and plumule length of mungbean seedlings but decreased both parameters when applied at $10 \%$ concentration. On the contrarily, stubble extract inhibited the growth of embryonic axes.

The increasing concentrations of oat root and stubble extracts decreased the fresh and dry weights of radical and plumule of mungbean seedlings, but increased the fresh and dry weights of cotyledons. The stubble extract proved to be more toxic than the root extract. Abu-Romman et al. (2010) reported that the radical and coleoptile length of the germinated seeds 
of wheat were significantly inhibited by the leaf leachate of Euphorbia hierosolymitana. Also, allelochemicals caused a significant reduction in root and shoot length, fresh weights, dry weights and decreased the amount of total chlorophyll and protein contents.

The recent trend in agriculture indicates to reduce tillage. This purposely maintains high amount of plant residues on soil surface and may cause many problems in the subsequently cultivation of crops. So, the present study was conducted to investigate the allelopathic effects of five selected weed species: Lambsquarters (Chenopodium album, L.), Petty squrge (Euphorbia peplus L.), Annual yellow sweet clover (Melilotus indica, (L.) All.), Wild oat (Avena fatua, L.) and Canary grass (Phalaris minor, Retz), on grain germination and seedling growth of wheat.

\section{MATERIALS AND METHODS}

Two experiments were performed in Agronomy Department, Faculty of Agriculture, Al-Azhar University, during 2008/2009 and 2009/2010 seasons. The first experiment was concerned with the effects of weeds water extracts (root and shoot) on germination and seedling growth of wheat under optimum conditions. The second experiment was to evaluate the effects of weed residues on emergence and seedling development of wheat in pots under wirehouse conditions.

Five winter weed species were selected (Table 1), to obtain plant material for residues and water extracts for germination test of wheat. These five weed species are common weeds in wheat fields in Egypt. The tested weeds were collected at flowering stage, transported to the laboratory and classified to roots and vegetative parts, air dried at room temperature, grounded to pass through $1 \mathrm{~mm}$ screen in Wiley mill and kept in plastic pages.

Table 1: The selected weed species that used in experiments

\begin{tabular}{|l|c|c|c|}
\hline No. & Family & Scientific name & English name \\
\hline 1 & Chenopodiaceae & Chenopodium album, L. & Lambsquarters or Goos foot \\
\hline 2 & Euphorbiaceae & Euphorbia peplus, L. & Petty squrge \\
\hline 3 & Fabaceae & Melilotus indica, (L.) All. & Annual yellow sweet clover \\
\hline \multirow{2}{*}{4} & \multirow{2}{*}{ Poaceae } & Avena fatua, L. & Wild oat \\
\cline { 3 - 4 } & & Phalaris minor, Retz. & Canary grass, Littleseed \\
\hline
\end{tabular}

The allelopathic effect of these weed species were tested against wheat (Triticum aestivum, L.) cv. Sakha 94. The seeds were kindly obtained from Field Crops Res. Inst., Agricultural Research Center, Ministry of Agriculture.

\section{Experiment (A): Effect of weed water extract on germination and seedling growth of wheat under optimum conditions.} Preparation of weeds extracts

Shoots and roots powders of five weed species were used to prepare water extract according to Abdallah et al. (1989) as follows; $25 \mathrm{~g}$ from each of root and shoot dried ground tissue were placed in $500 \mathrm{ml}$ Erlemeyer flask 
with $250 \mathrm{ml}$ of distilled water and mixture was shaked for $6 \mathrm{~h}$ on a horizontal shaker (approximately 160 cycles per minute). All extracts were filtered through cheese cloth to remove debris and finally filtered using (Whatman No. 1) filter paper to have $100 \%$ concentration $(100 \mathrm{~g} / \mathrm{L})$. The leachates of $10 \%\left(10 \mathrm{~cm}^{3} / \mathrm{L}\right), 20 \%\left(20 \mathrm{~cm}^{3} / \mathrm{L}\right)$ and $30 \%\left(30 \mathrm{~cm}^{3} / \mathrm{L}\right)$ concentrations were made by diluting the parent leachate with distilled water. All filtered water extracts were frozen in dark glass bottle for subsequent use. Crop grains were sanitized with sodium hypochloride solution $0.3 \%$ for 5 minutes before germination test. Petri- dished $(9 \mathrm{~cm}$ in diameter) were sterilized in autoclave at $121^{\circ} \mathrm{C}$ for 15 minutes and lined later Whatman No. 1 filter paper as a seed bed. The extracts concentrations $(0,10,20$ and $30 \%)$ of weed species parts evaluated on germination and growth of wheat seedlings. Fifteen-milliliter of each extract or of distilled water control were added to Petri dishes containing 25 grains of wheat crop. The Petri dishes were covered and placed in continuous dark in a controlled environment chamber which provided a constant temperature of $20^{\circ} \mathrm{C}$ according to ISTA (1996).

Petri dishes were arranged in completely randomized design with four replications per treatment. The parameters of this experiment were measured after 10 days from sowning .

\section{Experiment (B): Effect of weed residues on seedling development of wheat in pots under wirehouse conditions.}

The objective of this trial was to determine the liberation of allelochemicals from weed residues in soil and their influence on growth characters of seedling wheat. Ten grains of wheat were sown in 22 and 25 November at equal distances in experimental pots $(12 \mathrm{~cm}$ in diameter and 11 $\mathrm{cm}$ in height) filled with sand, previously washed carefully, mixed with the shoots ground material of each weed species residue at the rates of $0,1,2$ and 3\% (w/w). The seeds were sown on the surface and covered with additional mixed sand to give a final equal weight of each pot $(700 \mathrm{~g})$. The experiment was conducted in the wirehouse conditions. The pots were irrigated every two days with $100 \mathrm{ml}$ of tap water. The control pots were irrigated with the same water volume. Emerged seedling for each pot was recorded daily and seedlings were thinned to five seedlings per pot. Subsequently, new emerged seedling were recorded and thinned daily. However, data were recorded on selected seedling only and statistically analyzed. Seedling harvested after 45 days from sowing in two winter seasons of 2008/2009 and 2009/2010, respectively. Pots were arranged in a completely randomized design with four replications. At harvest date, seedlings of each pot were removed and washed carefully.

\section{Data recorded:}

1- Germination percentage (\%) (Expt.A) was recorded and calculated according to ISTA (1996).

2- Germination rate (Expt.A) was recorded at 1-day intervals and calculated as the following equation ISTA (1996). 
Germination rate

$$
=\frac{A_{1} \times B_{1}+A_{2} \times B_{2}+\ldots \ldots \ldots A_{n} \times B_{n}}{A_{1}+A_{2}+\ldots \ldots \ldots A_{n}}
$$

$A=$ number of germinated grains at the day of recording germinated grains $(B)$..

3- Root length $(\mathrm{cm})$.

4- Shoot length $(\mathrm{cm})$.

5- Number of green leaves/ plant (Expt.B).

6- Root fresh weight (g) (Expt.B).

7- Shoot fresh weight (g) (Expt.B).

8- Leaf area ( $\mathrm{cm}^{2} /$ plant) (Expt.B). Leaf area (LA) was determined according to the formula by Montgomery (1911),

$$
\text { Leaf area }=\mathrm{K} \times \mathrm{L} \times \mathrm{W}
$$

where: $\mathrm{K}=$ Adjustment factor (constant)

$\mathrm{L}=$ Length of leaf blade

$\mathrm{W}=$ Maximum width of leaf blade

The value of $K$ was 0.79 . The same formula was used by several researchers, e.g., Voldeng and Simpson (1967) and Aliu et al., (2010).

9- Root dry weight (mg).

10- Shoot dry weight (mg).

For dry weight determination, tissues were dried in an air forced oven at $70^{\circ} \mathrm{C}$ for $48 \mathrm{~h}$.

\section{Statistical analyses}

The data of each experiment were statistically analyzed as a factorial experiment according to the methods described by Little and Hills (1978). The combined analysis of the data of all experiments was followed both seasons (experiments), the treatment means were compared by Least Significant Differences (LSD) at the 0.05 level of probability.

\section{RESULTS AND DISCUSSION}

\section{Experiment (A): Effect of weed water extract on germination and seedling growth of wheat under optimum conditions.}

Results presented in Tables 2, 3 and 4 show means of the germination percentage, germination rate, root and shoot lengths as well as root and shoot weights of wheat seedling as affected by extracts of five weeds species. Results in Tables 2, 3 and 4 show that the differences between weed species for germination \%, germination rate, root length, shoot length, root dry weight and shoot dry weight were significant in the means of two seasons. Results indicated that Chenopodium album, L. extract significantly inhibited germination \% and germination rate, where Melilotus indica, (L.) All. extract significantly inhibited root length, shoot length, root dry weight and shoot dry weight compared with other weed species in the means of two seasons. This result may be due to inhibited effective for most the production of allelochemicals compounds in extraction. This result is in agreement with those of Qasem (1993 a\&b), Mallik et al. (1994), Kaletha et al. (1996) and Alam et al. (2002) since they found that lambsquarters Chenopodium album, L. was more allelopathicaly active weed. 
Osman, M. S. E. et al.

Table 2: Effect of weed species, weed parts and weed extract concentration levels (\%) on wheat grain germination percentage (\%) and germination rate after 10 days from sowing (means of two seasons).

\begin{tabular}{|c|c|c|c|c|c|c|c|c|c|c|c|}
\hline \multicolumn{2}{|c|}{ Treatment } & \multirow{2}{*}{\multicolumn{5}{|c|}{$\begin{array}{c}\text { Germination \% } \\
\begin{array}{c}\text { Concentration (C) } \\
\%\end{array}\end{array}$}} & \multirow{2}{*}{\multicolumn{5}{|c|}{$\begin{array}{c}\text { Germination rate } \\
\text { Concentration (C) } \\
\%\end{array}$}} \\
\hline \multirow{2}{*}{$\begin{array}{l}\text { Weed } \\
\text { species } \\
\text { (WS) }\end{array}$} & \multirow{2}{*}{$\begin{array}{l}\text { Weed } \\
\text { parts } \\
\text { (WP) }\end{array}$} & & & & & & & & & & \\
\hline & & 0 & 10 & 20 & 30 & Mean & 0 & 10 & 20 & 30 & Mean \\
\hline \multirow[b]{3}{*}{ W1 } & Root & 99.50 & 76.50 & 69.50 & 63.00 & 77.13 & 1.50 & 2.08 & 2.13 & 2.08 & 1.95 \\
\hline & Shoot & 99.50 & 50.50 & 33.38 & 21.00 & 51.10 & 1.50 & 2.55 & 2.70 & 2.76 & 2.38 \\
\hline & Mean & 99.50 & 63.50 & 51.44 & 42.00 & 64.11 & 1.50 & 2.32 & 2.42 & 2.42 & 2.17 \\
\hline \multirow[b]{3}{*}{ W2 } & Root & 99.50 & 87.00 & 85.00 & 76.50 & 87.00 & 1.50 & 1.37 & 1.37 & 1.51 & 1.44 \\
\hline & Shoot & 99.50 & 87.00 & 84.00 & 78.50 & 87.25 & 1.50 & 1.46 & 1.52 & 1.70 & 1.55 \\
\hline & Mean & 99.50 & 87.00 & 84.50 & 77.50 & 87.13 & 1.50 & 1.42 & 1.45 & 1.61 & 1.50 \\
\hline \multirow[b]{3}{*}{ W3 } & Root & 99.50 & 91.50 & 77.00 & 77.50 & 86.38 & 1.50 & 1.64 & 2.00 & 2.29 & 1.86 \\
\hline & Shoot & 99.50 & 59.00 & 36.00 & 19.00 & 53.38 & 1.50 & 2.30 & 2.46 & 2.80 & 2.27 \\
\hline & Mean & 99.50 & 75.25 & 56.50 & 48.25 & 69.88 & 1.50 & 1.97 & 2.23 & 2.55 & 2.07 \\
\hline \multirow[b]{3}{*}{ W4 } & Root & 99.50 & 89.00 & 84.00 & 80.00 & 88.13 & 1.50 & 1.35 & 1.43 & 1.48 & 1.44 \\
\hline & Shoot & 99.50 & 87.00 & 78.00 & 75.00 & 84.88 & 1.50 & 1.59 & 1.63 & 1.63 & 1.59 \\
\hline & Mean & 99.50 & 88.00 & 81.00 & 77.50 & 86.50 & 1.50 & 1.47 & 1.53 & 1.56 & 1.52 \\
\hline \multirow[b]{3}{*}{ W5 } & Root & 99.50 & 86.00 & 77.50 & 74.00 & 84.25 & 1.50 & 1.61 & 1.66 & 1.67 & 1.61 \\
\hline & Shoot & 99.50 & 79.50 & 72.00 & 66.00 & 79.25 & 1.50 & 1.74 & 1.87 & 1.87 & 1.75 \\
\hline & Mean & 99.50 & 82.75 & 74.75 & 70.00 & 81.75 & 1.50 & 1.68 & 1.77 & 1.77 & 1.68 \\
\hline \multirow{3}{*}{\begin{tabular}{|l} 
Mean \\
Mean \\
\end{tabular}} & Root & 99.50 & 86.00 & 78.60 & 74.20 & 84.58 & 1.50 & 1.61 & 1.72 & 1.80 & 1.66 \\
\hline & Shoot & 99.50 & 72.60 & 60.68 & 51.90 & 71.17 & 1.50 & 1.93 & 2.04 & 2.15 & 1.90 \\
\hline & & 99.50 & 79.30 & 69.64 & 63.05 & 77.88 & 1.50 & 1.77 & 1.88 & 1.98 & 1.78 \\
\hline \multicolumn{12}{|c|}{ LSD at $5 \%$ level } \\
\hline \multicolumn{3}{|c|}{ Weed species ( WS ) } & \multicolumn{4}{|r|}{1.78} & & & & & 0.09 \\
\hline \multicolumn{3}{|c|}{ Weed parts ( WP ) } & \multicolumn{4}{|r|}{1.13} & & & & & 0.06 \\
\hline \multicolumn{3}{|c|}{ Concentrations ( C ) } & \multicolumn{4}{|r|}{1.59} & & & & & 0.08 \\
\hline \multicolumn{3}{|c|}{ WS $\times W P$} & \multicolumn{4}{|r|}{2.52} & & & & & 0.13 \\
\hline \multicolumn{3}{|l|}{$\mathrm{WS} \times \mathrm{C}$} & \multicolumn{4}{|r|}{3.56} & & & & & 0.18 \\
\hline \multicolumn{3}{|c|}{$\mathrm{WP} \times \mathrm{C}$} & \multicolumn{4}{|r|}{2.25} & & & & & 0.11 \\
\hline \multicolumn{3}{|c|}{$W S \times W P \times C$} & \multicolumn{4}{|r|}{5.03} & & & & & N.S. \\
\hline
\end{tabular}

W1 = Chenopodium album, L., W2= Euphorbia peplus, L., W3= Melilotus indica, (L.) All., W4= Avena fatua, L., W5= Phalaris minor, Retz.

Concerning weed parts, the results in Tables 2, 3 and 4 show that the differences between weed parts extracted were significant for all studied traits in the means of two seasons. Results also, indicated that shoot extraction was more effective in its inhibitory effect than root extraction on all studied traits in the means of two seasons. The negative effect for the shoot extraction on studied traits may be attributed to increased allelochemicals compoounds to the harmful percentage in the shoot than the root. This results are in agreement with those of Qasem (1993a\&b) and El-Khatib et al. (2004), they found that the shoot extracts were inhibitory than root extracts.

With regard to extract concentrations, results indicate that the differences between extract concentrations were significant for all studieds character in the means of two seasons. The $30 \%$ concentration extract gave the highest inhibitory effect on germination percentage, germination rate, root and shoot 
lengths as well as root and shoot dry weights. Germination in distilled water gave the lowest germination rate, while the highest value was obtained from $30 \%$ concentation extract. Moreover, the concentrations 20 and $10 \%$ significantly reduced wheat grain germination and seedling growth. The effect of weed on studied attributes depends on weed extract according to Qasem (1993b). The interaction between weed species and weed parts was significant for all studied traits in the means of two seasons. The combination of Chenopodium album, L. with the shoot extract gave the highest inhibitetion value for germination \% and germination rate, where Melilotus indica, (L.) All. with the shoot extract gave the lowest value for root length, shoot length, root dry weight and the shoot dry weight in the means of two seasons.

Table 3: Effect of weed species, weed parts and weed extract concentration levels (\%) on wheat root and shoot length (cm) after 10 days from sowing (means of two seasons).

\begin{tabular}{|c|c|c|c|c|c|c|c|c|c|c|c|}
\hline \multicolumn{2}{|c|}{ Treatment } & \multirow{2}{*}{\multicolumn{5}{|c|}{$\begin{array}{c}\text { Root length (cm) } \\
\begin{array}{c}\text { Concentration (C) } \\
\%\end{array}\end{array}$}} & \multirow{2}{*}{\multicolumn{5}{|c|}{$\begin{array}{c}\text { Shoot length (cm) } \\
\text { Concentration (C) } \\
\%\end{array}$}} \\
\hline \multirow{2}{*}{\begin{tabular}{|c|} 
Weed \\
species \\
(WS)
\end{tabular}} & \multirow{2}{*}{$\begin{array}{c}\text { Weed } \\
\text { part } \\
\text { (WP) }\end{array}$} & & & & & & & & & & \\
\hline & & 0 & 10 & 20 & 30 & Mean & 0 & 10 & 20 & 30 & Mean \\
\hline \multirow{3}{*}{ W1 } & Root & \begin{tabular}{|l|}
22.25 \\
\end{tabular} & \begin{tabular}{|l|}
18.13 \\
\end{tabular} & 17.31 & 16.13 & 18.46 & 16.63 & 14.50 & 13.75 & 13.63 & 14.63 \\
\hline & Shoot & 22.25 & \begin{tabular}{|l|}
5.94 \\
\end{tabular} & 2.44 & 1.44 & \begin{tabular}{|l|}
8.02 \\
\end{tabular} & 16.63 & 13.63 & 12.25 & \begin{tabular}{|l|}
9.63 \\
\end{tabular} & 13.04 \\
\hline & Mean & \begin{tabular}{|l|}
22.25 \\
\end{tabular} & $\mid 12.04$ & \begin{tabular}{|l|}
9.88 \\
\end{tabular} & 8.79 & \begin{tabular}{|l|}
13.24 \\
\end{tabular} & 16.63 & \begin{tabular}{|l|}
14.07 \\
\end{tabular} & 13.00 & 11.63 & 13.83 \\
\hline \multirow{3}{*}{ W2 } & Root & 22.25 & 22.06 & 20.75 & 13.56 & 19.66 & 16.63 & 16.13 & 15.50 & 14.25 & 15.63 \\
\hline & Shoot & 22.25 & 15.88 & 13.63 & 8.31 & 15.02 & 16.63 & 14.75 & 14.38 & 14.25 & 15.00 \\
\hline & Mean & 22.25 & \begin{tabular}{|l|}
18.97 \\
\end{tabular} & 17.19 & 10.94 & 17.34 & 16.63 & 15.44 & 14.94 & 14.25 & 15.32 \\
\hline \multirow{3}{*}{ W3 } & Root & \begin{tabular}{|l|}
22.25 \\
\end{tabular} & \begin{tabular}{|l|}
13.81 \\
\end{tabular} & 11.19 & 9.19 & \begin{tabular}{|l|}
14.11 \\
\end{tabular} & 16.63 & 13.63 & 12.63 & 11.38 & 13.57 \\
\hline & Shoot & 22.25 & \begin{tabular}{|l|}
2.38 \\
\end{tabular} & 1.55 & 0.61 & \begin{tabular}{|l|}
6.70 \\
\end{tabular} & 16.63 & 8.88 & 6.00 & 3.13 & 8.66 \\
\hline & Mean & 22.25 & \begin{tabular}{|l|}
8.10 \\
\end{tabular} & \begin{tabular}{|l|l|}
6.37 \\
\end{tabular} & 4.90 & 10.41 & 16.63 & 11.26 & 9.32 & 7.26 & 11.11 \\
\hline \multirow{3}{*}{ W4 } & Root & 22.25 & 21.38 & 20.38 & 18.38 & 20.60 & 16.63 & 14.50 & 14.13 & 13.38 & 14.66 \\
\hline & Shoot & \begin{tabular}{|l|}
22.25 \\
\end{tabular} & 20.00 & 18.38 & \begin{tabular}{|l|}
17.56 \\
\end{tabular} & 19.55 & 16.63 & 15.63 & 15.25 & 14.25 & 15.44 \\
\hline & Mean & 22.25 & 20.69 & 19.38 & 17.97 & 20.07 & 16.63 & 15 & 14.69 & 1 & 15.05 \\
\hline \multirow{3}{*}{ W5 } & Root & 22.25 & 20.44 & 18.94 & 18.25 & 19.97 & 16.63 & 14.13 & 13.75 & 12.25 & 14.19 \\
\hline & Shoot & 22.25 & 16.69 & 13.19 & 8.19 & 15.08 & 16.63 & 13.75 & 13.25 & 13.00 & 14.16 \\
\hline & Mean & 22.25 & \begin{tabular}{|l|}
18.57 \\
\end{tabular} & 16.07 & 13.22 & 17.53 & 16.63 & 13.94 & 13.50 & 12.63 & 14.18 \\
\hline \multirow{2}{*}{ Mean } & Root & 22.25 & 19.16 & 17.71 & 15.10 & 18.56 & 16.63 & 14.58 & 13.95 & 12.98 & 14.53 \\
\hline & Shoot & 22.25 & 12.18 & 9.84 & 7.22 & 12.87 & 16.63 & 13.33 & 12.23 & 10.85 & 13.26 \\
\hline Mean & & 22.25 & $|15.67|$ & 13.78 & 11.16 & 15.72 & 16.63 & 13.96 & 13.09 & 11.92 & 13.90 \\
\hline \multicolumn{12}{|c|}{ LSD at $5 \%$ level } \\
\hline \multicolumn{3}{|c|}{ Weed species ( WS ) } & & & & 0.273 & & & & & 0.278 \\
\hline \multicolumn{3}{|c|}{ Weed parts (WP) } & & & & 0.173 & & & & & 0.176 \\
\hline \multicolumn{3}{|c|}{\begin{tabular}{|l|} 
Concentrations ( C ) \\
WS $\times$ WP
\end{tabular}} & & & & 0.244 & & & & & 0.249 \\
\hline \multirow{2}{*}{\multicolumn{3}{|c|}{$\frac{W S \times W P}{W S \times C}$}} & & & & 0.386 & & & & & 0.394 \\
\hline & & & & & & 0.546 & & & & & 0.557 \\
\hline \multicolumn{3}{|l|}{$W P \times C$} & & & & 0.345 & & & & & 0.352 \\
\hline \multicolumn{3}{|c|}{ WS $\times W P \times C$} & & & & 0.772 & & & & & 0.787 \\
\hline
\end{tabular}

The interaction of weed species and concentrations \% had a significant effect on all studied traits in the means of two seasons. The $30 \%$ concentration of Chenopodium album, L. extract recorded significantly 
Osman, M. S. E. et al.

inhibted for germination percentage as compared with all other treatments, while, the $30 \%$ concentration of Melilotus indica, (L.) All. extract gave the highest inhibition value for germination rate, root length, shoot length, root dry weight and the shoot dry weight in the means of two seasons as compared with all other treatments.

Table 4: Effect of weed species, weed parts and weed extract concentration levels (\%) on wheat root and shoot dry weight (mg) after 10 days from sowing (means of two seasons).

\begin{tabular}{|c|c|c|c|c|c|c|c|c|c|c|c|}
\hline \multicolumn{2}{|c|}{ Treatment } & \multirow{2}{*}{\multicolumn{5}{|c|}{$\begin{array}{c}\text { Root dry weight (mg) } \\
\text { Concentration (C) } \\
\%\end{array}$}} & \multirow{2}{*}{\multicolumn{5}{|c|}{$\begin{array}{c}\text { Shoot dry weight (mg) } \\
\text { Concentration (C) } \\
\%\end{array}$}} \\
\hline \multirow{2}{*}{\begin{tabular}{|l|} 
Weed \\
species \\
(WS)
\end{tabular}} & \multirow{2}{*}{\begin{tabular}{|l} 
Weed \\
part \\
(WP)
\end{tabular}} & & & & & & & & & & \\
\hline & & 0 & 10 & 20 & 30 & Mean & 0 & 10 & 20 & 30 & Mean \\
\hline \multirow{3}{*}{ W1 } & Root & 10.32 & \begin{tabular}{|l}
7.34 \\
\end{tabular} & 7.29 & \begin{tabular}{|l}
6.46 \\
\end{tabular} & \begin{tabular}{|l}
7.85 \\
\end{tabular} & 13.41 & 12.39 & 11.37 & 10.84 & 12.00 \\
\hline & Shoot & 10.32 & \begin{tabular}{|l|}
5.26 \\
\end{tabular} & 4.76 & \begin{tabular}{|l|}
2.29 \\
\end{tabular} & \begin{tabular}{|l|}
5.66 \\
\end{tabular} & 13.41 & \begin{tabular}{|l|l}
11.49 \\
\end{tabular} & 11.06 & 7.66 & 0.91 \\
\hline & Mean & 10.32 & \begin{tabular}{|l}
6.30 \\
\end{tabular} & 6.03 & \begin{tabular}{|l}
4.38 \\
\end{tabular} & \begin{tabular}{|l|l}
6.76 \\
\end{tabular} & 13.41 & 11.94 & 11.22 & 9.25 & 11.46 \\
\hline \multirow{3}{*}{ W2 } & Root & 10.32 & \begin{tabular}{|l|}
7.99 \\
\end{tabular} & 6.86 & \begin{tabular}{|l|}
5.96 \\
\end{tabular} & \begin{tabular}{|l}
7.78 \\
\end{tabular} & 13.41 & 111.51 & 10.30 & 9.20 & $\mid 11.11$ \\
\hline & Shoot & 10.32 & \begin{tabular}{|l|}
7.31 \\
\end{tabular} & 6.66 & \begin{tabular}{|l}
5.95 \\
\end{tabular} & \begin{tabular}{|l}
7.56 \\
\end{tabular} & 13.41 & & 10.35 & 9.66 & 11.20 \\
\hline & Mean & 10.32 & \begin{tabular}{|l|l|}
7.65 \\
\end{tabular} & 6.76 & \begin{tabular}{|l}
5.96 \\
\end{tabular} & \begin{tabular}{|l}
7.67 \\
\end{tabular} & 13.41 & 11.44 & 10.33 & 9.43 & 11.16 \\
\hline \multirow{3}{*}{ W3 } & Root & 10.32 & \begin{tabular}{|l|l}
6.73 \\
\end{tabular} & 6.40 & & \begin{tabular}{|l}
6.98 \\
\end{tabular} & 13.41 & & 9.35 & 9.25 & \begin{tabular}{|l|l}
10.67 \\
\end{tabular} \\
\hline & Shoot & 10.32 & \begin{tabular}{|l|}
6.05 \\
\end{tabular} & 5.12 & \begin{tabular}{|l|}
2.71 \\
\end{tabular} & 6.05 & 13.41 & \begin{tabular}{|l|}
8.48 \\
\end{tabular} & \begin{tabular}{|l}
7.67 \\
\end{tabular} & 3.77 & 8.33 \\
\hline & Mean & 10.32 & \begin{tabular}{|l|}
6.39 \\
\end{tabular} & 5.76 & \begin{tabular}{|l}
3.59 \\
\end{tabular} & \begin{tabular}{|l}
6.52 \\
\end{tabular} & 13.41 & 87 & 8.51 & 6.51 & 9.50 \\
\hline \multirow{3}{*}{ W4 } & Root & 10.32 & \begin{tabular}{|l}
8.99 \\
\end{tabular} & 8.49 & \begin{tabular}{|l}
8.10 \\
\end{tabular} & \begin{tabular}{|l|l}
8.98 \\
\end{tabular} & 13.41 & 10.51 & 9.80 & 9.48 & \begin{tabular}{|l|l}
10.80 \\
\end{tabular} \\
\hline & Shoot & 10.32 & \begin{tabular}{|l}
8.20 \\
\end{tabular} & 7.70 & \begin{tabular}{|l}
7.09 \\
\end{tabular} & \begin{tabular}{|l|l}
8.33 \\
\end{tabular} & 13.41 & 12 & 11.82 & 11.26 & 12.25 \\
\hline & Mean & 10.32 & \begin{tabular}{|l}
8.60 \\
\end{tabular} & 8.10 & $\begin{array}{l}7.60 \\
\end{array}$ & \begin{tabular}{|l|l}
8.65 \\
\end{tabular} & 13.41 & & 10.81 & 10.37 & 11.53 \\
\hline \multirow{3}{*}{ W5 } & Root & 10.32 & \begin{tabular}{|l}
8.71 \\
\end{tabular} & 8.52 & \begin{tabular}{|l}
8.41 \\
\end{tabular} & 8.99 & 13.41 & & 10.56 & 10.38 & 11.47 \\
\hline & Shoot & 10.32 & \begin{tabular}{|l|l}
8.55 \\
\end{tabular} & 8.17 & \begin{tabular}{|l|l}
8.04 \\
\end{tabular} & \begin{tabular}{|l|l}
8.77 \\
\end{tabular} & 13.41 & & 11.30 & 10.34 & 11.68 \\
\hline & Mean & 10.32 & \begin{tabular}{|l|}
8.63 \\
\end{tabular} & 8.35 & \begin{tabular}{|l|}
8.23 \\
\end{tabular} & \begin{tabular}{|l|}
8.88 \\
\end{tabular} & 13.41 & 11.60 & 10.93 & 10.36 & 11.58 \\
\hline \multirow{2}{*}{ Mean } & Root & 10.32 & \begin{tabular}{|l|}
7.95 \\
\end{tabular} & 7.51 & \begin{tabular}{|l}
6.68 \\
\end{tabular} & \begin{tabular}{|l}
8.12 \\
\end{tabular} & 13.41 & & 10.27 & 9.83 & $\mid$\begin{tabular}{|l|}
11.21 \\
\end{tabular} \\
\hline & Shoot & 10.32 & \begin{tabular}{|l|l}
7.07 \\
\end{tabular} & 6.48 & \begin{tabular}{|l|l}
5.21 \\
\end{tabular} & \begin{tabular}{|l|l}
7.27 \\
\end{tabular} & 13.41 & \begin{tabular}{|l|}
11.10 \\
\end{tabular} & 10.44 & 8.54 & \begin{tabular}{|l|l}
10.87 \\
\end{tabular} \\
\hline Mean & & 10.32 & \begin{tabular}{|l|l|} 
\\
\end{tabular} & 7.00 & 5.95 & \begin{tabular}{|l|l|}
7.70 \\
\end{tabular} & 13.41 & & 10.36 & 9.18 & 11.04 \\
\hline \multicolumn{12}{|c|}{ LSD at $5 \%$ level } \\
\hline \multicolumn{3}{|c|}{ Weed species (WS ) } & & & & 0.114 & & & & & 0.296 \\
\hline \multicolumn{3}{|c|}{ Weed parts (WP) } & & & & 0.072 & & & & & 0.18 \\
\hline \multirow{2}{*}{\multicolumn{3}{|c|}{ Concentrations ( C ) }} & & & & 0.102 & & & & & 0.265 \\
\hline & & $\mathrm{WS} \times \mathrm{WP}$ & & & & 0.161 & & & & & 0.41 \\
\hline \multirow{2}{*}{\multicolumn{3}{|c|}{$W S \times C$}} & & & & 0.227 & & & & & 0.592 \\
\hline & & $\mathrm{WP} \times \mathrm{C}$ & & & & 0.144 & & & & & 0.374 \\
\hline & & & & 0.321 & & & & & 0.837 \\
\hline
\end{tabular}

The interaction between weed parts and concentration\% was significant for all studied attributes in the means of two seasons. The shoot extract at $30 \%$ concentration gave the highest inhibition value for all studied traits.

Concerning the second order interaction of weed species $\times$ weed parts $\times$ concentration\%, results in Tables 2, 3 and 4 showed that, there was a significant effect on all studied traits, except germination rate. The combination Melilotus indica, (L.) All. $\times$ the shoot extraction $\times 30 \%$ con. 
significantly inhibted germination \%, root length, shoot length and the shoot dry weight, while, Chenopodium album, L. $\times$ the shoot extraction $\times 30 \%$ concentration significantly inhibted root dry weight in the means of two seasons. Also, results showed that, Melilotus indica, (L.) All. $\times$ the shoot extract $\times 30 \%$ concentration increased effective allelopathic under experiment conditions.

Experiment (B): Effect of weed residues on seedling development of wheat in pots under wirehouse conditions.

Results presented in Tables 5 and 6 showed that the differences between weed species for all studied criterias were significant. Chenopodium album, L. residuse was the highest inhibited root length, while Avena fatua, L. residuse significantly inhibited the shoot length, number of green leaves per plant, leaf area, shoot fresh and dry weights per plant in the means of two seasons. Euphorbia peplus, L. gave the highest inhibited the root dry weight per plant. However, Melilotus indica, (L.) All. residuse significantly inhibited the root fresh weight in the means of two seasons. These results were obtained by Bhowmik and Doll (1982) and El-Khatib et al. (2004).

Results in Tables 5 and 6 also indicated that the residues concentrations had significant effect on all studied traits the means of two seasons. The $3 \%$ concentration residuse gave the highest inhibitory effect for all criteria as compared with all other concentrations (Tables 5 and 6). These results are in agreement with Qasem (1993b) and Kawisi et al. (1995).

From the results in Tables 5 and 6 data showed that weed species had significant effect on concentration\% as factor in means of two seasons, this means that the interaction between Melilotus indica, (L.) All. with $3 \%$ concentration gave the highest inhibition value for root fresh weight, Avena fatua, L. with $3 \%$ concentration significantly inhibitation the shoot fresh weight, shoot dry weight, shoot length, no. of green leaves and leaf area per plant, while, Euphorbia peplus L. and seem con. gave the highest value for root dry weight, as well as, Chenopodium album, L. with $3 \%$ concentration recorded the highest value for the root length in means of two seasons.

Results confirmed the phenomenon of allelopathy and its marked sharing in weed-crop interaction. Chenopodium album, L., Melilotus indica, (L.) All. and Avena fatua, L. were the highest dengerous weeds in our weed interference trials. The highest dangerous weeds in weed interference trials were imposed also the strengther allelopathic impact on germination and seedling growth of wheat. In addition weed residues may be phytotoxic and greatly reduced germination and establishment of wheat seedling. 
Osman, M. S. E. et al.

Table 5: Effect of weed species, weed residues concentration levels (\%) and their interaction on wheat root and shoot length $(\mathrm{cm})$, number of green leaves and leaf area $\left(\mathrm{cm}^{2}\right)$ after 45 days from sowing (means of two seasons).

\begin{tabular}{|c|c|c|c|c|c|}
\hline \multicolumn{2}{|c|}{ Treatment } & \multirow[b]{2}{*}{$\begin{array}{c}\text { Root length } \\
(\mathrm{cm})\end{array}$} & \multirow[b]{2}{*}{$\begin{array}{c}\text { Shoot } \\
\text { length }(\mathrm{cm})\end{array}$} & \multirow[b]{2}{*}{$\begin{array}{l}\text { No. of } \\
\text { green } \\
\text { leaves }\end{array}$} & \multirow[b]{2}{*}{$\begin{array}{l}\text { Leaf area } \\
\qquad\left(\mathrm{cm}^{2}\right)\end{array}$} \\
\hline $\begin{array}{l}\text { Weed } \\
\text { species } \\
\text { (WS) }\end{array}$ & \begin{tabular}{|c|}
$\begin{array}{c}\text { Weed residues } \\
\text { concentration } \\
\text { (C) } \%\end{array}$ \\
\end{tabular} & & & & \\
\hline \multirow{5}{*}{ W1 } & 0 & 31.49 & 37.86 & 5.44 & 40.95 \\
\hline & 1 & 21.09 & 35.09 & 5.28 & 38.25 \\
\hline & 2 & 14.39 & 32.79 & 5.08 & 31.11 \\
\hline & 3 & 11.03 & 28.24 & 4.63 & 22.40 \\
\hline & Mean & 19.50 & 33.50 & 5.11 & 33.18 \\
\hline \multirow{5}{*}{ W2 } & 0 & 31.49 & 37.86 & 5.44 & 40.95 \\
\hline & 1 & 23.26 & 32.28 & 4.44 & 24.10 \\
\hline & 2 & 15.14 & 27.14 & 3.90 & 15.85 \\
\hline & 3 & 13.59 & 22.16 & 3.60 & 13.90 \\
\hline & Mean & 20.87 & 29.86 & 4.35 & 23.70 \\
\hline \multirow{5}{*}{ W3 } & 0 & 31.49 & 37.86 & 5.44 & 40.95 \\
\hline & 1 & 21.19 & 30.78 & 4.48 & 23.48 \\
\hline & 2 & 15.21 & 26.93 & 4.13 & 20.13 \\
\hline & 3 & 12.19 & 24.89 & 3.68 & 15.30 \\
\hline & Mean & 20.02 & 30.12 & 4.43 & 24.97 \\
\hline \multirow{5}{*}{ W4 } & 0 & 31.49 & 37.86 & 5.44 & 40.95 \\
\hline & 1 & 28.61 & 26.19 & 3.24 & 14.35 \\
\hline & 2 & 24.70 & 24.11 & 2.96 & 13.26 \\
\hline & 3 & 21.71 & 22.20 & 2.81 & 11.41 \\
\hline & Mean & 26.63 & 27.59 & 3.61 & 19.99 \\
\hline \multirow{5}{*}{ W5 } & 0 & 31.49 & 37.86 & 5.44 & 40.95 \\
\hline & 1 & 24.79 & 36.40 & 4.90 & 38.49 \\
\hline & 2 & 19.89 & 33.04 & 4.36 & 27.81 \\
\hline & 3 & 18.19 & 28.81 & 3.65 & 20.86 \\
\hline & Mean & 23.59 & 34.03 & 4.59 & 32.03 \\
\hline \multirow{5}{*}{$\begin{array}{c}\text { Concentration } \\
\%\end{array}$} & 0 & 31.49 & 37.86 & 5.44 & 40.95 \\
\hline & 1 & 23.79 & 32.14 & 4.47 & 27.73 \\
\hline & 2 & 17.87 & 28.80 & 4.09 & 21.63 \\
\hline & 3 & 15.34 & 25.26 & 3.67 & 16.77 \\
\hline & Mean & 22.12 & 31.02 & 4.42 & 26.77 \\
\hline \multicolumn{2}{|c|}{ LSD at $5 \%$ level } & & & & \\
\hline \multicolumn{2}{|c|}{ Weed species ( WS ) } & 1.26 & 0.81 & 0.14 & 1.23 \\
\hline \multicolumn{2}{|c|}{ Concentrations ( C ) } & 1.13 & 0.73 & 0.13 & 1.10 \\
\hline \multicolumn{2}{|c|}{$\mathrm{WS} \times \mathrm{C}$} & 2.53 & 1.63 & 0.28 & 2.46 \\
\hline
\end{tabular}

W1= Chenopodium album, L., W2= Euphorbia peplus, L., W3= Melilotus indica, (L.) All., W4= Avena fatua, L., W5= Phalaris minor, Retz. 
Table 6: Effect of weed species, weed residues concentration levels (\%) and their interaction on wheat root and shoot weight after 45 days from sowing (means of two seasons).

\begin{tabular}{|c|c|c|c|c|c|}
\hline \begin{tabular}{|r} 
Trea \\
$\begin{array}{c}\text { Weed species } \\
\text { (WS) }\end{array}$
\end{tabular} & $\begin{array}{l}\text { Concentration } \\
\text { (C) \% }\end{array}$ & $\begin{array}{l}\text { Root fresh } \\
\text { weight }(\mathrm{g})\end{array}$ & $\begin{array}{c}\text { Shoot fresh } \\
\text { weight }(g)\end{array}$ & $\begin{array}{c}\text { Root dry } \\
\text { weight (mg) }\end{array}$ & $\begin{array}{c}\text { Shoot dry } \\
\text { weight (mg) }\end{array}$ \\
\hline & 0 & 1.53 & 1.23 & 156.41 & 141.12 \\
\hline & 1 & 1.05 & 1.03 & 86.55 & 134.64 \\
\hline W1 & 2 & 0.71 & 0.87 & 54.74 & 111.76 \\
\hline & 3 & 0.51 & 0.65 & 42.17 & 78.87 \\
\hline & Mean & 0.95 & 0.95 & 84.97 & 116.60 \\
\hline & 0 & 1.53 & 1.23 & 156.41 & 141.12 \\
\hline & 1 & 0.70 & 0.58 & 64.87 & 70.39 \\
\hline W2 & 2 & 0.65 & 0.43 & 47.55 & 55.65 \\
\hline & 3 & 0.54 & 0.38 & 32.96 & 43.36 \\
\hline & Mean & 0.86 & 0.66 & 75.45 & 77.63 \\
\hline & 0 & 1.53 & 1.23 & 156.41 & 141.12 \\
\hline & 1 & 0.63 & 0.64 & 61.51 & 79.29 \\
\hline W3 & 2 & 0.53 & 0.52 & 49.10 & 67.83 \\
\hline & 3 & 0.38 & 0.41 & 40.24 & 59.49 \\
\hline & Mean & 0.77 & 0.70 & 76.82 & 86.93 \\
\hline & 0 & 1.53 & 1.23 & 156.41 & 141.12 \\
\hline & 1 & 1.15 & 0.36 & 119.51 & 53.79 \\
\hline W4 & 2 & 1.03 & 0.31 & 95.90 & 47.24 \\
\hline & 3 & 0.87 & 0.28 & 77.46 & 41.74 \\
\hline & Mean & 1.15 & 0.55 & 112.32 & 70.97 \\
\hline & 0 & 1.53 & 1.23 & 156.41 & 141.12 \\
\hline & 1 & 1.45 & 1.17 & 146.34 & 136.80 \\
\hline W5 & 2 & 1.21 & 0.75 & 110.47 & 96.94 \\
\hline & 3 & 1.02 & 0.46 & 82.94 & 66.03 \\
\hline & Mean & 1.30 & 0.90 & 124.04 & 110.22 \\
\hline & 0 & 1.53 & 1.23 & 156.41 & 141.12 \\
\hline & 1 & 0.99 & 0.76 & 95.76 & 94.98 \\
\hline$\underset{\%}{\text { Concentration }}$ & 2 & 0.82 & 0.57 & 71.55 & 75.88 \\
\hline & 3 & 0.66 & 0.44 & 55.15 & 57.90 \\
\hline & Mean & 1.00 & 0.75 & 94.72 & 92.74 \\
\hline LSD at $5 \%$ leve & & & & & \\
\hline Weed species & (WS ) & 0.052 & 0.050 & 6.02 & 4.00 \\
\hline Concentrations & $5(\mathrm{C})$ & 0.046 & 0.045 & 5.36 & 3.58 \\
\hline$W S \times C$ & & 0.103 & 0.100 & 12.04 & 8.01 \\
\hline
\end{tabular}

W1 = Chenopodium album, L., W2= Euphorbia peplus, L., W3= Melilotus indica, (L.) All., W4= Avena fatua, L., W5= Phalaris minor, Retz.

\section{REFERENCES}

Abdallah, M.M.F.; C.L. Elmore and K.A. Khalaf (1989). Allelopathic effects of tomato root extract on the germination of some weed seed. Egypt. J. Hort., 16 (1): 25-33. 
Abu-Romman, S.; M. Shatnawi and R. Shibli (2010). Allelopathic effects of spurge (Euphorbia hierosolymitana) on wheat (Triticum durum, L.). American-Eurasian J. Agric. \& Environ. Sci., 7 (3): 298-302.

Alam, S.M.; M.A. Khan; S.M. Mujtaba and A. Shereen (2002). Influence of aqueous leaf extract of common lambsquarters and $\mathrm{NaCl}$ salinity on the germination, growth, and nutrient content of wheat. Acta Physiologiae Plantarum, 24 (4): 359-364.

Aliu, S.; Sh. Fetahu and L. Rozman (2010). Variation of physiological traits and yield components of some maize hybrid (Zea mays, L.) in agroecological conditions of Kosovo. Acta Agric. Slovenica, 95: (1): 35-41.

Bhowmik, P.C. and J.D. Doll (1982). Corn and soybean response to allelopathic effects of weed and crop residues. Agron. J., 74: 601-606.

Boonitiee, A. and P. Ritdhit (1984). Allelopathic effects of some weeds on mungbean plants (Vigna radiata). Proc. $1^{\text {st }}$ tropical weed science, cont, vol. 2. oct. 22-25. Jat. Yai, songkwa Thailand: 401-406.

El-Khatib, A.A.; A.K. Hegazy and Hanaa K. Galal (2004). Does allelopathy have a role in the ecology of Chenopodium murale? Ann. Bot. Fennici., 41: 37-45.

Hucl, P. (1998). Response to weed control by four spring wheat genotypes differing in competitive ability. Canadian J. Plant Sci., 78(1): 171-173.

I.S.T.A. (1996). International Seed Testing Association. Seed Sci. \& Technol., 24, Supplement, Rules. Pp: 155-183.

Kaletha, M.S.; B.P. Bhatt and N.P. Todaria (1996). Allelopathic crop weed interactions in traditional agroforestry systems of Gerhwal Himalaya. Allelopathy J., 3(1): 65-70.

Kaushalya, G.; J. Veena; I.S. Solanki and Tulika (2005). Effect of aqueous extracts of root and stubble of oat (Avena sativa, L.) on seedling growth and protein utilization in mungbean (Vigna radiata, L.). Allelopathy $\mathrm{J}$. 16(2): 279-287. [C.F. CAB Abst. 2006/01-2006/11, AN: 20063129027].

Kawisi, M.; O.A. Chivinge and A.B. Mashingaidze (1995). Allelopathic effects of purple nutsedge (Cyperus rotundus, L.) tuber extracts on germination and seedling growth of some horticultural crops in Zimbabwe. Zimbabwe J. of Agric. Res., 33(1): 117-131.[ C.F. Hort. Abst. 69: 3964].

Keeley, P.E. (1987). Interference and interaction of purple and yellow nutsedge (Cyperus rotundus and Cyperus esculentus) with crops. Weed Technology, 1(1): 74-81.

Little, T.M. and F.G. Hills (1978). Agricultural and experimentation. John Wily \& Sons Inc., New York Pp. 31-52.

Löve, A. (1984). Conspectus of the Triticaceae. Feddes Repertorium, 95: 425 521.

Mallik, M.A.; R. Puchala and F.A. Grosz (1994). A growth inhibitory factor from lambsquarters (Chenopodium album, L.). J. Chem. Ecol., 20(4): 957-967. [C.F. CAB Abst. 1993-4/1995, AN: 952302110].

Montgomery, E.C. (1911). Correlation studies in corn. Annual Report No. 24. Nebraska Agric. Exp. Stn. Lincoln, NE, Pp. 108-159.

Nisha, C.; S. Harpal; H.P. Tripahi; N. Chopra and H. Singh (1999). Critical period of weed crop competition in wheat (Triticum aestivum, L.). Indian J. Weed Sci., 31(3-4): 151-154. 
Qasem, J.R. (1993a). Allelopathic effect of nettle-leaved goosefoot (Chenopodium murale) on wheat and barley. Dirasat Series B Pure and Applied Sci., 20(1): 80-94.

Qasem, J.R. (1993 b). Allelopathic effect of some common weeds on growth of wheat and barley. Dirasat. Series B Pure and Applied Scie., 20(2): 5-28. [C.F. CAB Abst. 1993-4/1995, AN: 942303368].

Rice, E.L. (1974). Allelopathy. Academic press, New York. An update Bot. Rev. 45: 15-109.

Voldeng, H.D. and G.M. Simpson (1967) : Leaf area as an indicator of potential grain yield in wheat. Con. J. Plant Sci., 47: 359-365.

التأثير الأليلوبـاثى المحتمل لخمسـة أنواع من الحثـائش على إنبات ونمو بـادرات

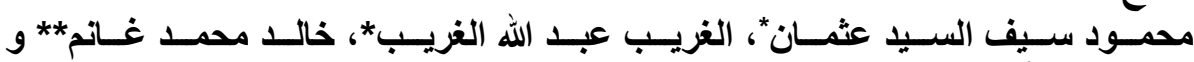

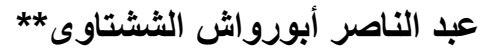

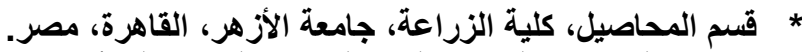

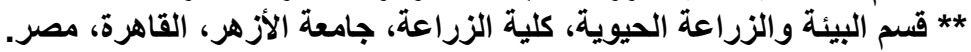

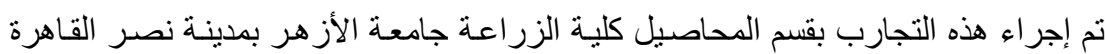

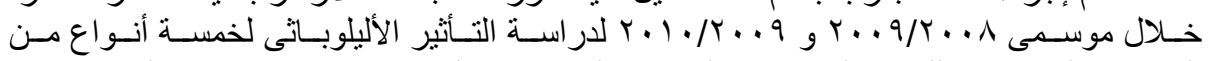

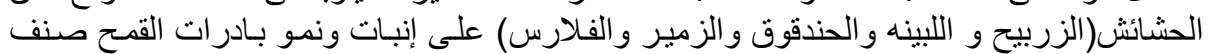

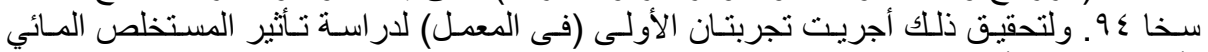

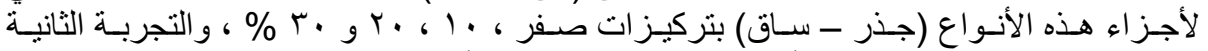

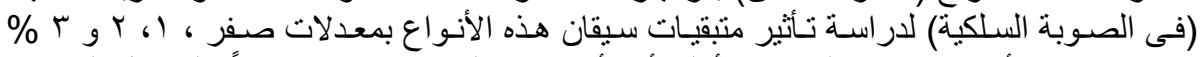

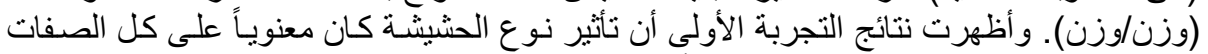

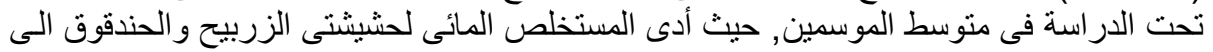

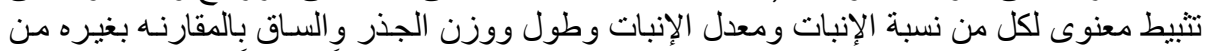

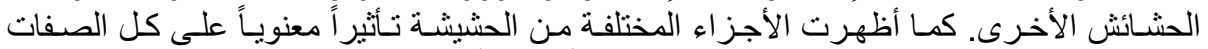

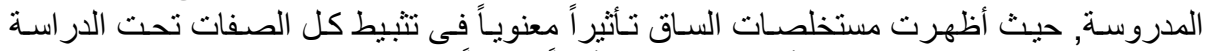

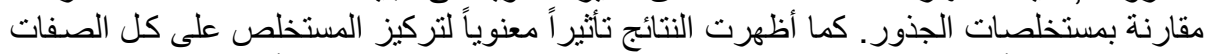

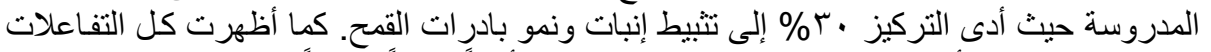

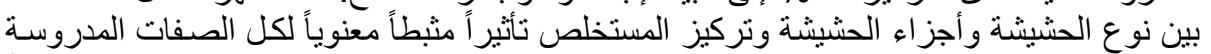

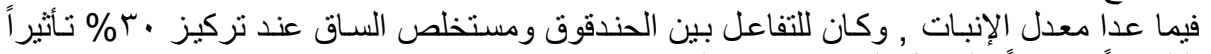

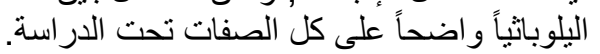

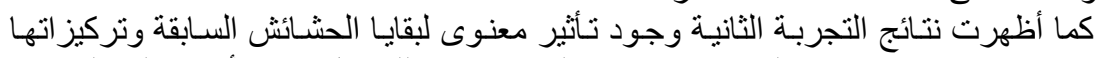

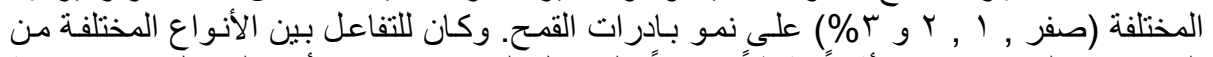

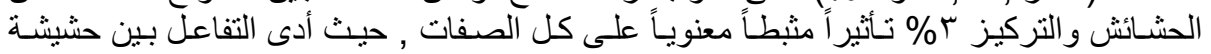

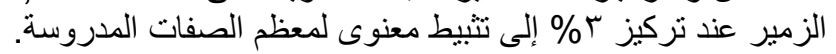

كلية الزراعة - جامعة المنصورة كلية زراعة مشتهر - جامعة جامعة بنها

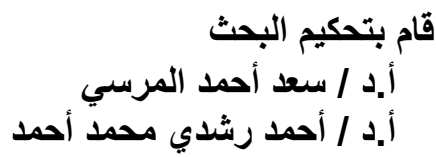

